EPJ Web of Conferences 61, 04018 (2013)

DOI: $10.1051 /$ epjconf/ 20136104018

(C) Owned by the authors, published by EDP Sciences, 2013

\title{
Multiwavelength Observations of 6 BL Lac Objects in 2008-2012
}

\author{
D.A. Morozova1 ${ }^{1}$, S.G. Jorstad ${ }^{1,2}$, A.P. Marscher², V.M. Larionov¹, I.S. Troitskiy ${ }^{1}$, D.A. Blinov ${ }^{3,1}$, I. Agudo ${ }^{4,5,2}$, and \\ P.S. Smith ${ }^{6}$ \\ ${ }^{1}$ Astron. Inst., St.-Petersburg State Univ., Russia e-mail: comitcont@gmail.com \\ ${ }^{2}$ Institute for Astrophysical Research, Boston University, MA, USA \\ ${ }^{3}$ University of Crete, Heraklion, Greece \\ ${ }^{4}$ Joint Institute for VLBI in Europe, Dwingeloo, Netherlands \\ ${ }^{5}$ Instituto de Astrofisíca de Andalucía, CSIC, Granada, Spain \\ ${ }^{6}$ Steward Observatory, University of Arizona, Tucson, AZ, USA
}

\begin{abstract}
We present results of 4 years of VLBA monitoring along with $\gamma$-ray and optical R-band photometric observations of 6 BL Lac objects (3C 66A, S5 0716+71, PKS 0735+17, S4 0954+68, W Com, and OT 081). We have analyzed total intensity images obtained with the VLBA at $43 \mathrm{GHz}$ and investigated the kinematic evolution of the parsec scale jets of the sources. For all sources we compare flux variations in the VLBI core and bright superluminal knots with $\gamma$-ray and optical light curves. The majority of $\gamma$-ray flares have optical counterparts. $67 \%$ of the $\gamma$-ray events are coincident with the appearance of new superluminal knots and/or flares in the millimeter-wave core. These results support the conclusion that for many flares in blazars the region of the $\gamma$-ray and optical emission is located in the vicinity or downstream of the mm-wave VLBI core.
\end{abstract}

\section{Introduction}

Blazars display high variability at different timescales over a broad range of frequencies. Their extreme properties are thought to be the result of their relativistic jets pointing toward us. Although blazars comprise only a few percent of the overall AGN population, they represent the most numerous class of objects identified with $\gamma$-ray sources. The origin of this high-energy radiation is still not clear, although according to radio-interferometer observations $\gamma$ ray bright blazars have the most relativistic jets [1, 2]. There are a number of studies that have revealed a connection between the $\gamma$-ray emission and jet properties (e.g. [3-5]).

\section{Observations and data reduction}

We obtain optical (R-band) flux densities from photometric observations at the $0.4 \mathrm{~m}$ telescope of St. Petersburg State University (LX200) and $0.7 \mathrm{~m}$ telescope of the Crimean Astrophysical Observatory (AZT-8). The data analysis for these telescopes is described in [6]. We also use R-band data carried out with the Perkins and Liverpool Telescopes (the BU group ${ }^{1}$ ), Calar Alto Telescopes ${ }^{2}$, and Steward Observatory ${ }^{3}$.

We derive 0.1-200 GeV $\gamma$-ray flux densities by analyzing data from the Large Area Telescope (LAT) of the

\footnotetext{
${ }^{1}$ http://www.bu.edu/blazars/VLBAproject.html

${ }^{2}$ http://www.iaa.es/ iagudo/research/MAPCAT/MAPCAT.html

${ }^{3}$ http://james.as.arizona.edu/ psmith/Fermi/
}

Fermi Gamma-ray Space Telescope with the standard software [7]. We have constructed $\gamma$-ray light curves with binning from 1 to 7 days (depending on the source's brightness), with a detection criterion that the maximumlikelihood test statistic (TS) should exceed 10.0.

We use total intensity radio images at $43 \mathrm{GHz}$ obtained with the Very Long Baseline Array $\left(\mathrm{VLBA}^{1}\right)$. We have modelled the images in terms of a small number of components with circular Gaussian brightness distributions. The core is a stationary feature located at one of the ends of the portion of the jet that is visible at $43 \mathrm{GHz}$. Identification of components in the jet across epochs is based on analysis of their flux, position angle, distance from the core, and size. We have computed kinematic parameters of knots (the proper motion, velocity, and acceleration) by fitting the positions of a component over epochs by different polynomials of order from 1 to 4 in the same manner as described in [8].

\section{Observational results and discussion}

Fig. 1 presents (from top to bottom) the light curves at radio wavelengths ( $7 \mathrm{~mm}$ VLBI-core), the R-band optical light curves, and the Fermi LAT $\gamma$-ray light curves (orange circles correspond to upper limits) of 6 blazars during the period from MJD 54600 to MJD 56000 (from 2008 August to 2012 August). Fig. 2 shows the VLBA images of the sources at $43 \mathrm{GHz}$. We have examined VLBA images of the sources for both variability of the core and appearance of superluminal knots ejected during this period. Table 1 

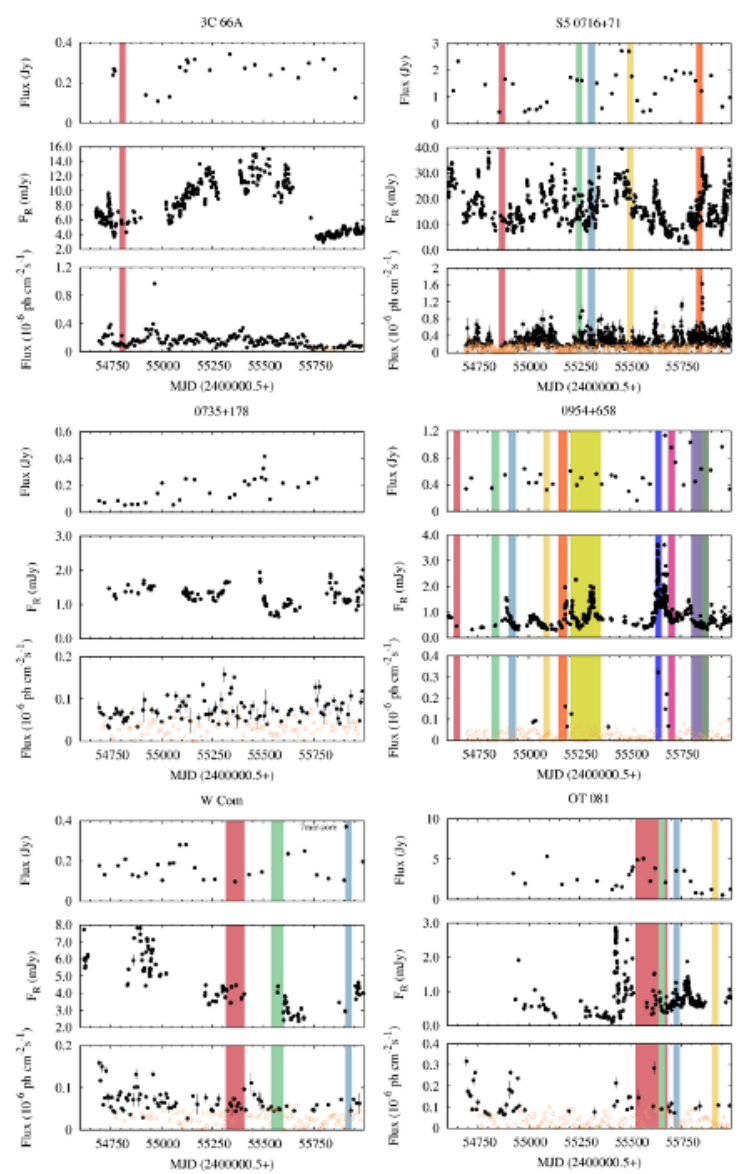

Figure 1. From top to bottom: evolution of the flux at radio wavelengths, R-band optical and $\gamma$-ray light curves (orange points mark upper limits), during 2008-2012. Multicolor vertical areas correspond to the time of ejection of a knot, with the width of each area equal to $\pm 1 \sigma$ uncertainty of $T_{\text {eject }}$.

lists the apparent speed of moving knots, $\beta_{a p p}$, acceleration, if detected, $\left(\dot{\mu}_{\|}\right.$and $\dot{\mu}_{\perp}$, along and perpendicular to the jet, respectively), mean position angle with respect to the core, $\langle\Theta\rangle$, time of separation from the core, $T_{\text {eject }}$, and time of the peak of a $\gamma$-ray flare, $T_{\max }$, if the latter occurred within $2 \sigma$ uncertainty of $T_{\text {eject }}$.

We have detected both moving and stationary components in all 6 objects (see Fig. 3). We have detected 13 stationary features located within a range of projected distances of 0.5-3.4 pc from the core. According to [3] stationary hot spots are a common characteristic of compact jets. Sources 3C 66A, S5 0716+71, PKS 0735+17, S4 0954+68, W Com, and OT 081 displayed 1, 5, 2, 10, 3, and 4 moving knots, respectively. All sources are bright enough to be detected routinely at $\gamma$-ray energies, except S4 0954+68, which is marginally detected with 7 day binning.

All sources are variabile from radio to optical wavebands. The source S5 $0716+71$ exhibits the highest $\gamma$-ray activity during 2008-2012, however all the other sources show substantial variability in $\gamma$-rays as well. The $\gamma$-ray activity coincides with an increase of the total intensity in

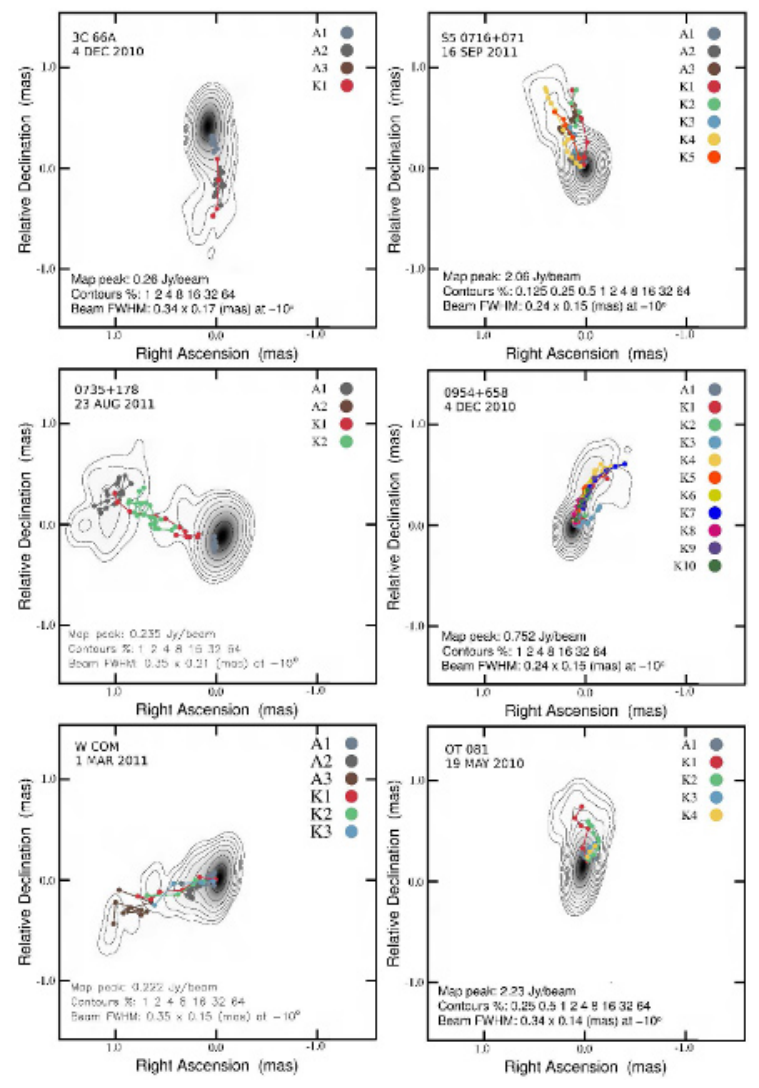

Figure 2. VLBA images of the sources with trajectories of the knots superposed.

the core at $43 \mathrm{GHz}(7 \mathrm{~mm})$ and the appearance of superluminal knots in the jet.

3C 66A: The light curve shows increasing $\gamma$-ray flux of the object during MJD $54700-55750$ (Fig. 1). The source dislpays a high level of optical flux and an increase of the radio flux during this period. A new superluminal knot K1 passed through the mm-wave core after the second $\gamma$-ray flare (MJD 54805). In addition, the source shows an increase of the flux level at optical and radio wavelengths during MJD 55000 - 55800 in the form of a plateau. We have identified 3 stationary features at the distances of $\sim 0.17, \sim 0.58$ and $\sim 2.35$ mas from the core.

S5 0716+71: Persistent activity is apparent across the electromagnetic spectrum (Fig. 1). We have identified 5 moving superluminal knots K1-K5 (Fig. 3), out of which 3 components emerged into the jet during the high optical and $\gamma$-ray state. We have identified 3 stationary features at distances of $\sim 0.06, \sim 0.13$ and $\sim 0.5$ mas from the core. As we have reported in [9], knot K5 appeared at MJD $\sim 55850$, which coincides within $1 \sigma$ uncertainty with the time of the highest peak in the $\gamma$-ray light curve. This event is accompanied by a rotation of the position angle of optical polarization (EVPA) by $180^{\circ}$. In addition, we have found a change in the jet direction from $\sim 11^{\circ}$ (in 2008-2010, knots K1 and K2) to $\sim 36^{\circ}$ (in late 2010-2012, knots K3-K5). During the $\gamma$-ray flares at MJD $~ 55627$ 
and $~ 55757$ no moving components were detected, but the flux of the $7 \mathrm{~mm}$ core increased significantly (Fig.1).

PKS 0735+17: We have detected 2 moving knots in PKS 0735+17 (Fig. 3), which were ejected earlier than MJD 54600, and two stationary features at $\sim 0.1$ and $\sim$ 1.2 mas from the core. However, the source was quiescent over the period of observations at $\gamma$-ray energies and also at optical and $7 \mathrm{~mm}$ wavelengths.

S4 0954+68: The light curve in Fig. 1 shows high activity of the source in the optical band beginning at MJD 54900, with a number of strong flares. We have identified 10 superluminal knots (Fig. 3), out of which 9 (K2-K10) components emerged into the jet during the high optical state. Also, we have detected one stationary feature at the distance $\sim 0.2$ mas from the core. Although the $\gamma$-ray flux was below the detection limit during most of the period of our observations, there are a number of positive detections during the strong optical flares that were contemporaneous with the passages of knots through the core. The ejection of knots $\mathrm{K} 7$ and $\mathrm{K} 8$ coincides within $1 \sigma$ uncertainty with the major flare in the R-band light curve and detections at $\gamma$-ray energies. As we have reported in [10], the appearance of knot K7 was accompanied by a significant rotation, $\sim 300^{\circ}$, of the optical EVPA. All components lie at nearly the same position angle relative to the core, $\sim-20^{\circ}$, except component $\mathrm{K} 3$, for which $<\Theta>\sim-53^{\circ}$.

W Com: We observe 3 moving knots, K1 - K3 (Fig. 3), in this blazar. A new superluminal knot, K1, passed through the mm-wave core before the $\gamma$-ray flare (MJD 55442), although the ejection occurred within $2 \sigma$ uncertainty of $T_{\text {eject }}$ with respect to the flare peak. We have identified three stationary features at distances of $\sim 0.14, \sim 0.29$ and $\sim 0.93$ mas from the core.

OT+081: The light curve (Fig.1) shows high activity of the source at optical and radio wavelengths. Although the source is usually quite faint at $\gamma$-ray energies, there are a number of $\gamma$-ray flares that appear to be associated with flares at optical and radio wavelengths. We detect 4 moving knots, K1-K4, which were ejected during the period of our observations. We have found a stationary component at a distance of $\sim 0.1$ mas from the core. The trajectory of knot K2 is significantly curved (Fig.2). The time of the passage of knots K1, K2, and K3 through the mm-wave core coincides with the $\gamma$-ray and optical events (Table ).

\section{Summary}

Over the period from August 2008 to August 2012 we detected superluminal motion in the parsec scale jets of all 6 objects, with apparent speeds ranging from $4 \mathrm{c}$ to $30 \mathrm{c}$. We have found that a high level of the $\gamma$-ray activity in 5 out of the 6 blazars studied coincides with the appearance of a new superluminal knot and/or a flare in the millimeterwave core, as well as optical flares. The remaining source, PKS $0735+17$, was quiescent at $\gamma$-ray energies and at $\mathrm{mm}$ wavelengths during the study. Our preliminary analysis finds that 10 out of $15 \gamma$-ray flares were simultaneous with
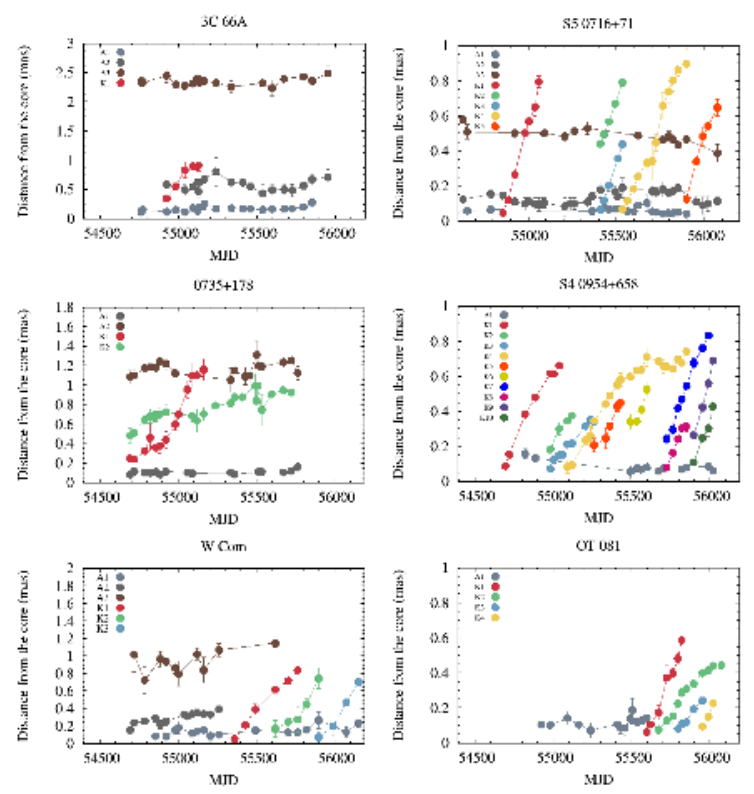

Figure 3. Separations of the knots from the core as a function of time.

the passage of a superluminal knot through the core within the errors of $T_{\text {eject }}$ or with a major outburst in the core at 7 $\mathrm{mm}$. We have detected 14 superluminal knots with no apparent connection with $\gamma$-ray activity. However, 9 of these events can be associated with optical flares. These results support the conclusions that $\gamma$-ray and optical flares in blazars are cospatial, and that many of these flares are located in the vicinity or downstream of the mm-wave VLBI core. However, the data do not exclude that some events can be produced closer to the central engine.

\section{Acknowledgements}

We thank M.Aller for access to the UMRAO data. We also acknowledge The Owens Valley Radio Observatory (OVRO) for online-data access. The Submillimeter Array is a joint project between the Smithsonian Astrophysical Observatory and the Academia Sinica Institute of Astronomy and Astrophysics, and is funded by the Smithsonian Institution and the Academia Sinica.The research at St.Petersburg State University was partly funded by RFBR grant 12-02-31193 and 12-02-00452. The VLBA is operated by the National Radio Astronomy Observatory. The National Radio Astronomy Observatory is a facility of the National Science Foundation, operated under cooperative agreement by Associated Universities, Inc. The Boston University group was partly funded by NASA Fermi Guest Investigator grants NNX08AV65G, NNX11AQ03G, and NNX11AO37G. Paul Smith acknowledges funding support from NASA Fermi Guest Investigator grants NNX08AW56G and NNX09AU93G. I. Agudo acknowledges funding support from the Spanish Ministry of Economy and Competitiveness and the Regional Government of Andalucia grants AYA2010-14844 and P09-FQM-4784. 


\section{References}

[1] T. Savolainen, D.C. Homan, T. Hovatta, M. Kadler, Y.Y. Kovalev, M.L. Lister, E. Ros, J.A. Zensus, A\&A 512, A24 (2010), 0911. 4924

[2] Y.Y. Kovalev, H.D. Aller, M.F. Aller, D.C. Homan, M. Kadler, K.I. Kellermann, Y.A. Kovalev, M.L. Lister, M.J. McCormick, A.B. Pushkarev et al., ApJ 696, L17 (2009), 0902 . 2085

[3] S.G. Jorstad, A.P. Marscher, J.R. Mattox, A.E. Wehrle, S.D. Bloom, A.V. Yurchenko, ApJS 134, 181 (2001), arXiv:astro-ph/0101570

[4] A.P. Marscher, S.G. Jorstad, V.M. Larionov, M.F. Aller, H.D. Aller, A. Lähteenmäki, I. Agudo, P.S. Smith, M. Gurwell, V.A. Hagen-Thorn et al., ApJ 710, L126 (2010), 1001 . 2574

[5] A.P. Marscher, S.G. Jorstad, F.D. D'Arcangelo, P.S. Smith, G.G. Williams, V.M. Larionov, H. Oh, A.R. Olmstead, M.F. Aller, H.D. Aller et al., Nature 452, 966 (2008)
[6] V.M. Larionov, S.G. Jorstad, A.P. Marscher, C.M. Raiteri, M. Villata, I. Agudo, M.F. Aller, A.A. Arkharov, I.M. Asfandiyarov, U. Bach et al., A\&A 492, 389 (2008), 0810. 4261

[7] W.B. Atwood, A.A. Abdo, M. Ackermann, W. Althouse, B. Anderson, M. Axelsson, L. Baldini, J. Ballet, D.L. Band, G. Barbiellini et al., ApJ 697, 1071 (2009), 0902. 1089

[8] S.G. Jorstad, A.P. Marscher, M.L. Lister, A.M. Stirling, T.V. Cawthorne, W.K. Gear, J.L. Gómez, J.A. Stevens, P.S. Smith, J.R. Forster et al., AJ 130, 1418 (2005), arXiv: astro-ph/0502501

[9] V.M. Larionov, S.G. Jorstad, A.P. Marscher, D.A. Morozova, D.A. Blinov, V.A. Hagen-Thorn, T.S. Konstantinova, E.N. Kopatskaya, L.V. Larionova, E.G. Larionova et al., ApJ 768, 40 (2013), 1303.2218

[10] V.M. Larionov, D.A. Morozova, I.S. Troitsky, D.A. Blinov, E.N. Kopatskaya, E.G. Larionova, S.G. Jorstad, A.P. Marscher, Fermi Symposium proceedings - eConf C110509 (2011), 1110.5861

Table 1. Parameters of the knots

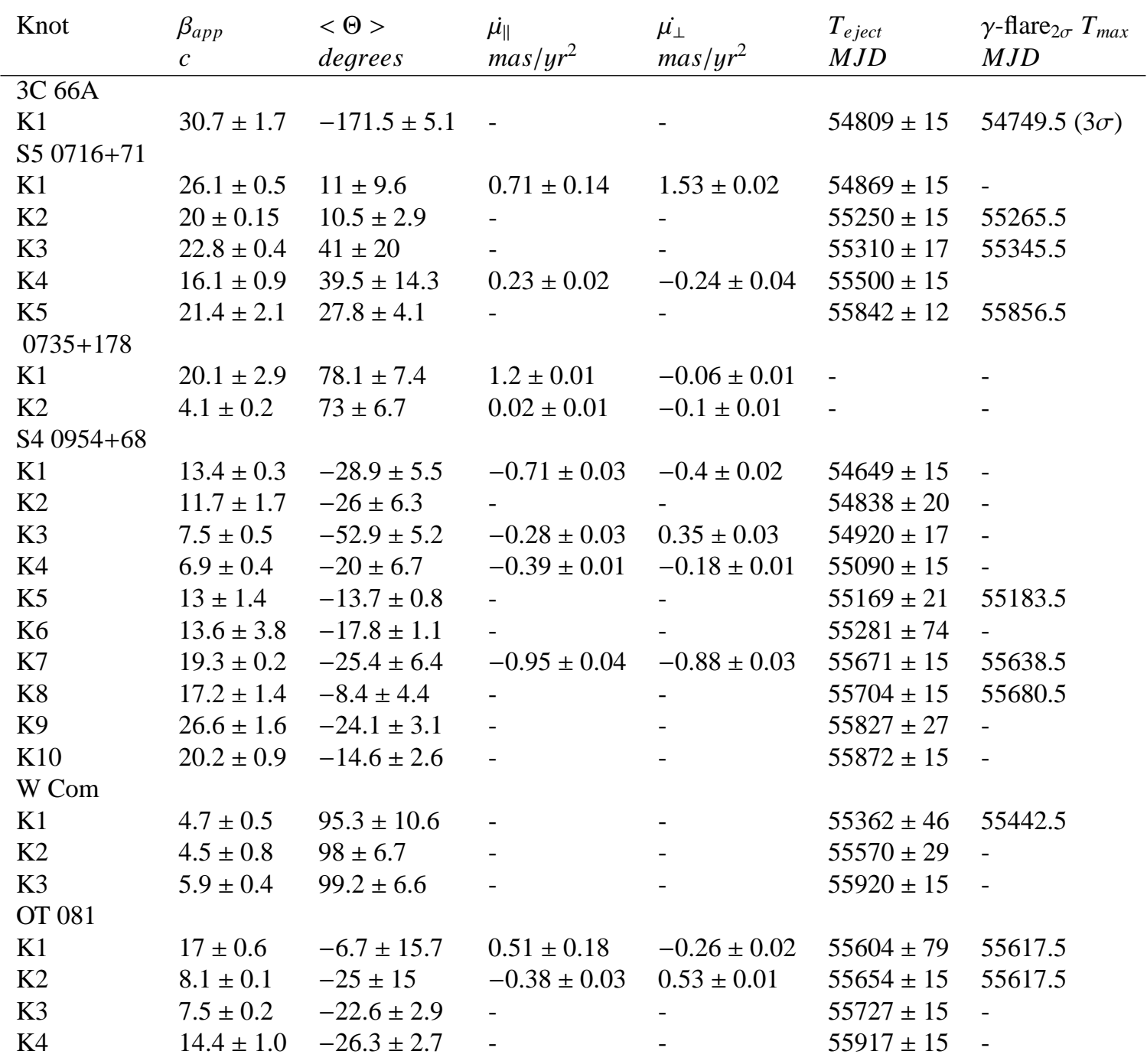

